\title{
ANNIVERSARY \\ On the 70th Anniversary of Birth of Sergey Borisovich Bulgarevich
}

Received September 1, 2011

DOI: $10.1134 / \mathrm{S} 107036321111034 \mathrm{X}$

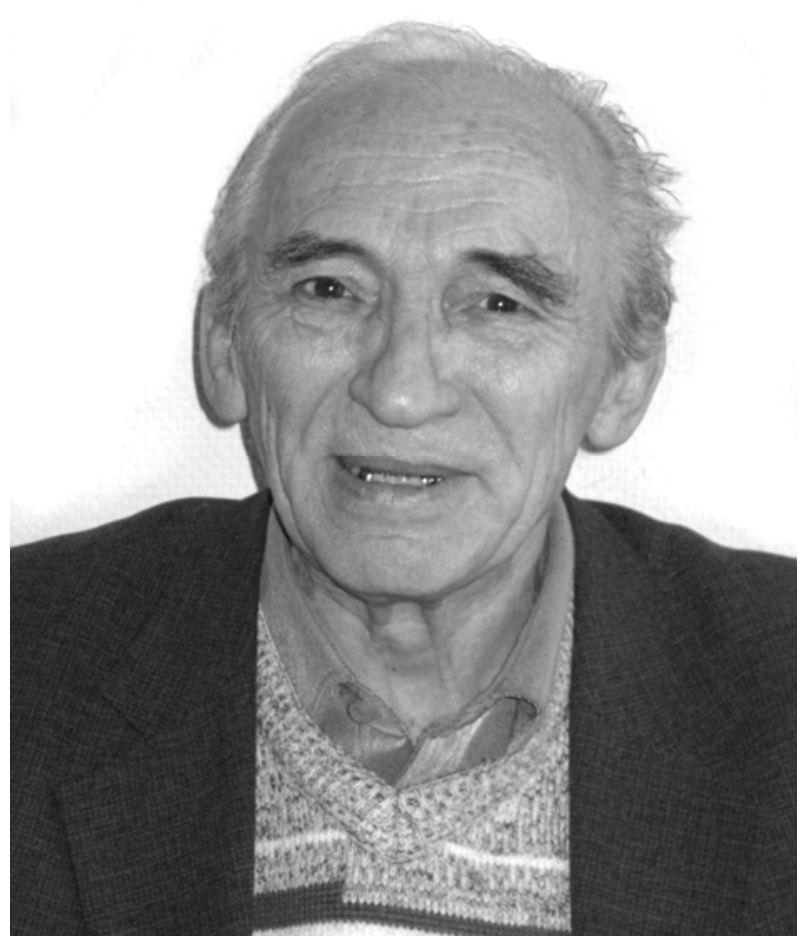

On September 4, 2011 the prominent scientist, Doctor of Chemical Sciences, Professor Sergey Borisovich Bulgarevich celebrated his 70-year-old anniversary.

In 1966 S.B. Bulgarevich graduated from the Krasnodar Institute of Chemical Technology by the speciality "Chemical Technology of Plastics" and received the qualification of engineering chemist. He continued education as the postgraduate student at the Chair of Physical and Colloidal Chemistry of the Rostov State University. In 1973 he successfully defended a candidate thesis entitled "Research of the Kerr Effect in Molecular Complexes," and in 1985, a doctoral thesis entitled "Molecular Polarizability, Polarity, and Steric Structure of Heteroaromatic Compounds and Their Complexes." In 1988 S.B. Bulgarevich was given the rank of professor on the speciality "Physical Chemistry."

In 1980-2001 S.B. Bulgarevich worked as the Head of laboratory "Electric and Magnetic Methods of Research of Molecular Structure" and fulfilled functions of the Deputy Head of the department "Structure and Catalytic Properties of Coordination Compounds" at the Scientific Research Institute of Physical and Organic Chemistry at the Rostov State University. He was a member of the Scientific Councils on the awarding of Candidate and Doctor degrees at the Rostov State University.

Professor S.B. Bulgarevich is a known scientist and an acknowledged expert in Russia and abroad in the field of research on the structure and properties of organic and complex compounds in solutions by means of electro- and magnetooptical physical methods and methods of dipole moments and molar volumes. He performed a number of works in cooperation with the leading research centers in Russia, Ukraine, Italy, Germany, and Spain.

At present S.B. Bulgarevich works at the Rostov State University of Communication Means as the Professor of the chair "Chemistry." Sergey Borisovich is an author of more than 270 scientific works. One doctoral and 11 candidate dissertations were successfully fulfilled and defended under his supervision.

Friends, disciples, and colleagues cordially congratulate Sergey Borisovich with the 70th anniversary of birth and wish him a sound health, happiness, and new scientific achievements. 